Check for updates

Cite this: RSC Adv., 2017, 7, 23954

\title{
Coalescence of droplets on micro-structure patterned hydrophobic planar solid surfaces
}

\author{
Guiping Zhu, ${ }^{a}$ Hui Fan, ${ }^{\text {b Hulin Huang }}{ }^{\mathrm{a}}$ and Fei Duan ${ }^{* b}$
}

The motion and coalescence of sessile liquid droplets on patterned solid surfaces are investigated systematically in terms of the liquid and substrate surface properties. A patterned silicon surface is decorated with circular pillars. The fabrication of the substrate is carried out by following standard photolithography. A thin photoresist layer of AZ-9260 on the pillars makes the surface exhibit superhydrophobic properties. The pillars are obtained using a deep reactive-ion etching process. Due to the deposited material and the increment of surface roughness, the liquid wetting behavior varies dramatically. The wetting properties of deionized water droplets on the patterned surface are examined to improve the understanding of the coalescence process. The coalescence of droplets is realized using capillary force on one of the droplets sitting at the boundary of surfaces with and without micro-patterns.

Received 21st March 2017

Accepted 7th April 2017

DOI: 10.1039/c7ra03323k

rsc.li/rsc-advances

application. For example, superhydrophobic surfaces are gaining increasing interest due to potential applications such as self-cleaning windows, nanofluidic devices, superhydrophobic (repelling dirt/water) fabrics, and anti-icing strategies. ${ }^{22,23}$ Condensed water microdroplets are demonstrated to exhibit self-propelled jumping behavior when introducing microporearray nanostructures on solid substrates. ${ }^{24,25}$ In addition to the nanostructure equipped superhydrophobic surfaces, coating with an aqueous lubricating layer can also provide adhesion reduction on the solid substrates, which is much more efficient for anti-icing or anti-fogging applications. ${ }^{26-28}$ The induced motion inception results in coalescence behavior of liquid droplets. Controllable droplet coalescence also provides an important approach to realize reactions within droplets. ${ }^{29}$ In the early stages, considerable efforts have been devoted to research on the coalescence of droplets floating in a liquid medium..$^{30,31}$ As the development of applications in a wide variety of fields continues, numerous studies have been dedicated to researching the characteristics of coalescence between two droplets. Qian and $\mathrm{Law}^{32}$ showed that a permanent coalescence would be realized only if the Weber number is smaller than a critical value, which depends on the impact parameter $B=\chi /(2 R)$, with $\chi$ representing the distance between the centers of the droplets along the moving direction, and $R$ being the radius of the equalsized droplets. The attainment of coalescence is further found relating to the surroundings, surface tension, and viscosity of the droplets.

Rapid coalescence can be achieved through manipulating discrete droplets inside micro channels, ${ }^{33,34}$ on confined plates, ${ }^{35}$ or on solid substrates with hydrophobic properties. However, the investigation of coalescence between discrete droplets dispensed onto a solid surface has received remarkable attention for important practical applications. Two stages are

${ }^{a}$ College of Astronautics, Nanjing University of Aeronautics
Street, Qinhuai District, Nanjing, Jiangsu, China 210016

${ }^{b}$ School of Mechanical and Aerospace Engineering, Nanyang Technological University, 50 Nanyang Avenue, Singapore 639798.E-mail:feiduan@ntu.edu.sg 
mentioned during the coalescence process of sessile droplets. The first stage, which is an initial formation of a meniscus bridge, has been extensively explored for its significant importance in applications where the droplets totally wet the surfaces. Based on lubrication theory, the theoretical governing equation is derived for the evolution of the resulting thin film upon coalescence without considering the gravitational effect. ${ }^{36}$ In addition, Diez and Kondic ${ }^{37}$ proposed a computational method for conducting a three-dimensional thin film flow including surface tension as well as gravity forces. Recently, the volume-of-fluid method was applied in a numerical simulation of the generation of the meniscus bridge for coalescence between a pair of equal-size sessile droplets. ${ }^{38}$ The second stage, which is the kinetics of droplets' coalescence on a solid surface, can be examined experimentally and theoretically. ${ }^{39,40}$ In addition to the critical factors for the freely-suspended droplets, the motion of the triple contact line was found to be strongly prominent for coalescence kinetics of sessile droplets. ${ }^{40}$ An experimental study was implemented to reveal the coalescence dynamics of droplets sitting on hydrophobic surfaces. ${ }^{41}$ The extensive effects of the initial conditions and surface properties were also investigated. ${ }^{\mathbf{4 2}}$

Various surface modifications can be achieved using microfabrication technology, and hence different wetting characteristics on surfaces can be controllable. The micro-structured surfaces are applicable for wettability change and motion of liquid droplets based on specific designs. Therefore, it is important to study the properties of the patterned surfaces through liquid wetting studies. Due to the self-propelled behavior under special wettable conditions, we aim to realize droplet coalescence on the boundary of superhydrophobic and hydrophilic substrates. As far as we know, the kinematics of droplets under these circumstances have not been discussed systematically in terms of the microstructure design, droplet size, etc. In this paper, we present the surface treatment using standard lithography followed by deep reactive-ion etching (DRIE) to produce three different substrates fabricated on silicon wafers. Based on the understanding of liquid wettability on the patterned surfaces, rapid droplet coalescence can be realized with one droplet approaching another stationary one from the wettability boundary. This is potentially applicable in mixing and coalescence for chemical and biochemical assays. We systematically present the droplet distribution during the whole process. Furthermore, the kinematics of the droplet are discussed on the basis of the design of the substrates and the approaching droplet size. In the experiments, one of the droplets was dispensed at the boundary of two regions (one region is flat while the other region is patterned). Instead of using external active sources such as electric and magnetic forces, the movement and coalescence of droplets were produced by the capillary force on the droplet.

\section{Materials and experiments}

A silicon substrate was used for the experiments. The polished surface of the substrate was decorated with arrays of circular pillars at different diameters and interval sizes. Due to the limit of wet etching in achieving homogeneous treatment, the height of the pillars was considered to be at the value of around 100 $\mu \mathrm{m}$. The time and velocity for etching was set to be the same in order to obtain identical heights of the pillars. As a result, the effect of pillar height was not examined in this work. However, the wettability of the liquid droplet on the structured surface always follows the Cassie-Baxter model.

Fig. 1(a) shows the detailed procedure for the fabrication using soft lithography. AZ-9260 was spin coated onto a cleaned 4-inch silicon wafer. It was followed by the soft baking process, where the AZ-9260 layer was hardened onto the silicon substrate (step 1). The configuration of pillars was designed and printed on a $5 \times 5$ inch plastic film. The mask was then used to carry out the UV exposure (step 2). Subsequently, an AZ-400K/water solution was used for the development of the AZ-9260 mold (step 3). In step 4, a deep reactive-ion etching process was performed to form pillars with heights around the value of $100 \mu \mathrm{m}$. Focused views of the different specimens fabricated on silicon wafers are presented in Fig. 1(b)-(d). The specimens in Fig. 1(b)-(d) are named I, II, and III in order to facilitate the following description and discussion. The characterizations of I, II, and III are listed in detail in Table 1 . They have increasing pillar intervals from $25 \mu \mathrm{m}$ to $100 \mu \mathrm{m}$, but share the same pillar diameter and height at $50 \mu \mathrm{m}$ and $100 \mu \mathrm{m}$, respectively.

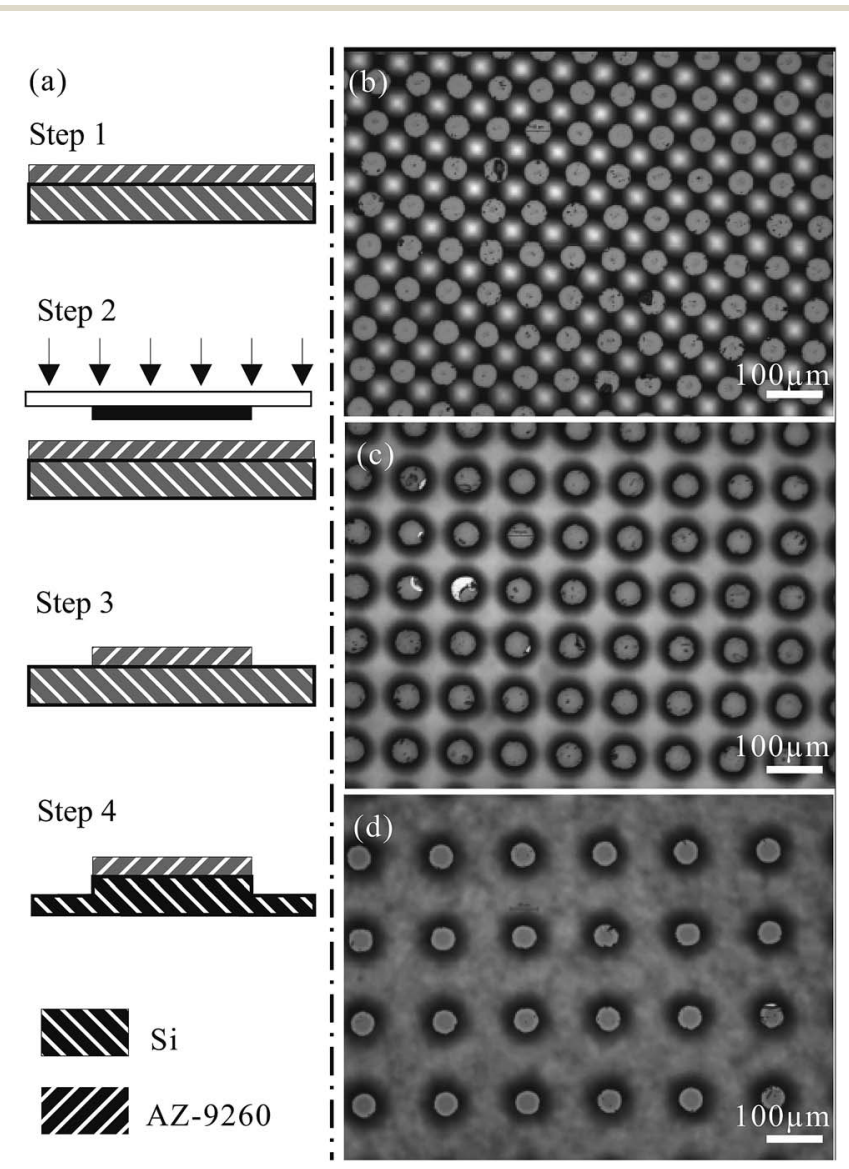

Fig. 1 Fabrication of microchannels with soft lithography. (a) The fabrication process (step 1: AZ-9260 spin coated on a silicon wafer; step 2: UV exposure with a mask; step 3: development of AZ-9260 mold; step 4: DRIE). (b) Image of specimen I, (c) image of specimen II, and (d) image of specimen III. 
Table 1 Characterizations of the pillars in specimens I, II, and III

\begin{tabular}{llll}
\hline Specimen & Height $(\mu \mathrm{m})$ & Diameter $(\mu \mathrm{m})$ & $\begin{array}{l}\text { Interval } \\
(\mu \mathrm{m})\end{array}$ \\
\hline I & 100 & 50 & 25 \\
II & 100 & 50 & 50 \\
III & 100 & 50 & 100
\end{tabular}

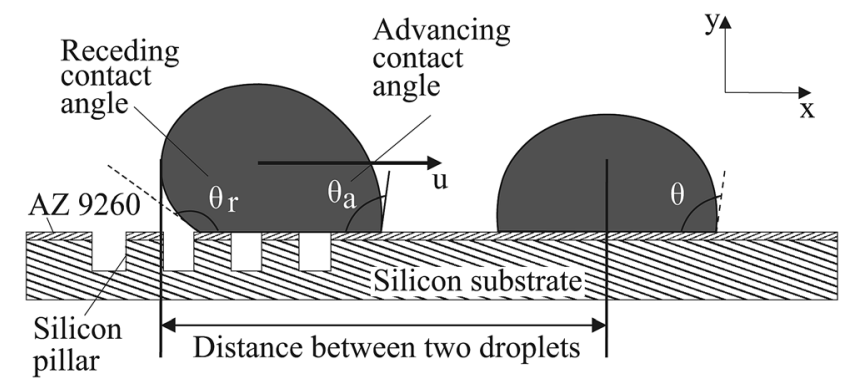

Fig. 2 The schematic setup for the capillary-induced droplet motion.

Mili-Q grade II deionized (DI) water was used to prepare the droplet. The droplet shape can be estimated to be spherical when the Bond number, Bo, is $<1$, where Bo $=\Delta \rho g L^{2} / \sigma$ in which $\Delta \rho, g, L$, and $\sigma$ indicate density difference, gravitational acceleration, characteristic length, and surface tension, respectively. In order to get a small Bond number $($ Bo $<1)$, the size of the liquid droplet dispensed was controlled. The kinematics of the droplets were investigated by droplet self-propulsion under capillary force. A micropipette (Finnpipette F1, Thermo Scientific, United States) is capable of dispensing droplets with different volumes ranging from $1 \mu \mathrm{L}$ to $10 \mu \mathrm{L}$. In the experiment shown in Fig. 2, a droplet is dispensed on the boundary that

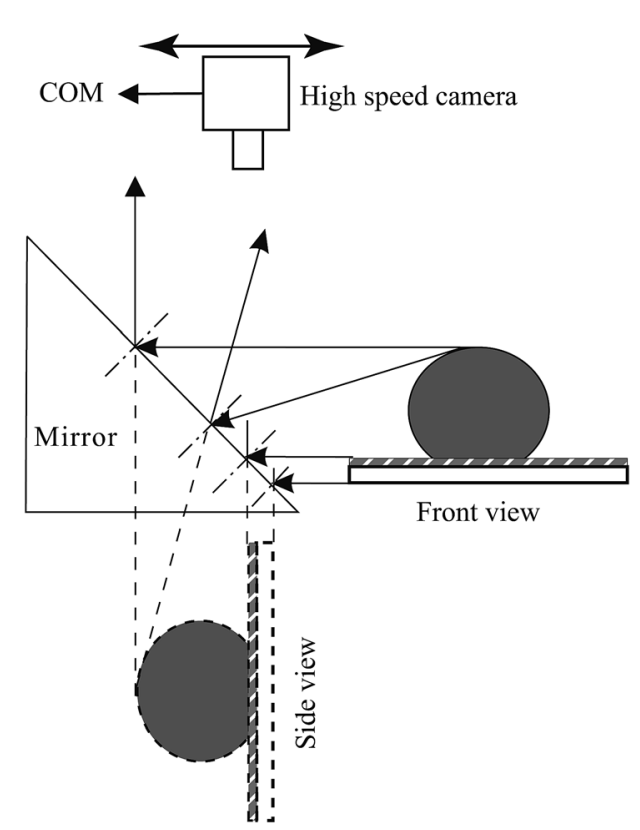

Fig. 3 Schematic diagram of the imaging system that could provide both top and side views at the same time. distinguishes the portions with and without micro-patterns. There should be a wettability gradient between the two sides. Consequently, the force balance on the triple contact line of the droplet is broken up. The resultant capillary force drives the droplet to move towards the side without micro-patterns. The coalescence of the two droplets can be achieved and this can potentially be used for chemical and biochemical assays.

The experimental setup is shown in Fig. 3. A high speed camera is mounted on a mounting stand rotated by 90 degrees, with the lens pointing downward. A 45 degree prism mirror is placed close to the water droplet that is dispensed onto the specimen. The 45 degree prism mirror is able to reflect the side view of the water droplet to the camera, hence enabling the camera to obtain both the top and side view of the water droplet reaction on the specimen at the same time. Through the use of a pipette, the volume of the droplets can be precisely controlled to maintain consistency. Calibration in the experiments is carried out using a ruler inserted into the image region.

\section{Results}

The capillary force induced motion of droplets sitting on the transition of hydrophobic and hydrophilic surfaces is proven to be a success on all three of the specimens investigated. The droplet sitting on the transition line is observed to gain the capillary force to break itself off the tip of the pipette and move completely to the side with the hydrophilic surface.

The experiment is carried out with a fixed volume stationary droplet of $5 \mu \mathrm{L}$ and approaching droplets of $5 \mu \mathrm{L}, 7 \mu \mathrm{L}$, and $9 \mu \mathrm{L}$. The stationary droplet is dispensed onto the silicon wafer surface adjacent to the modified surface while the second droplet is dispensed onto the transition line. Fig. 4-6 show the side views of a $5 \mu \mathrm{L}$ droplet merging with a second $5 \mu \mathrm{L}$ droplet on specimens I, II, and III.

In order to quantify the movement and coalescence efficiency based on the capillary-induced droplet motion, the distances between two droplets are plotted in Fig. 7. The distance is measured from the center line of the stationary droplet to the end of the moving droplet, as shown in Fig. 2. The moving droplet moves towards the stationary one after it detaches from the pipette tip. For specimen I, with the lowest pillar interval, the distance between the droplets reduces the slowest and the spreading of the resultant merged droplet is strongest of the three specimens. For specimen III, with the highest pillar interval, the distance between the droplets reduces more quickly and the spreading of the resultant merged droplet is weakened. These results agree well with our previous discussion suggesting a larger pillar interval will result in the surface of the specimen having stronger hydrophobicity. As a result, the droplet coalescence on specimen III is the fastest.

The capillary force on the approaching droplet depends on droplet base diameter, water droplet surface tension, and advancing and receding contact angles. The base diameter of a droplet mainly depends on the liquid volume dispensed on the superhydrophobic substrate. Fig. 8-10 illustrate the distance between two droplets on all of the specimens with the volume of the approaching droplet varying from $5 \mu \mathrm{L}$ to $9 \mu \mathrm{L}$. 


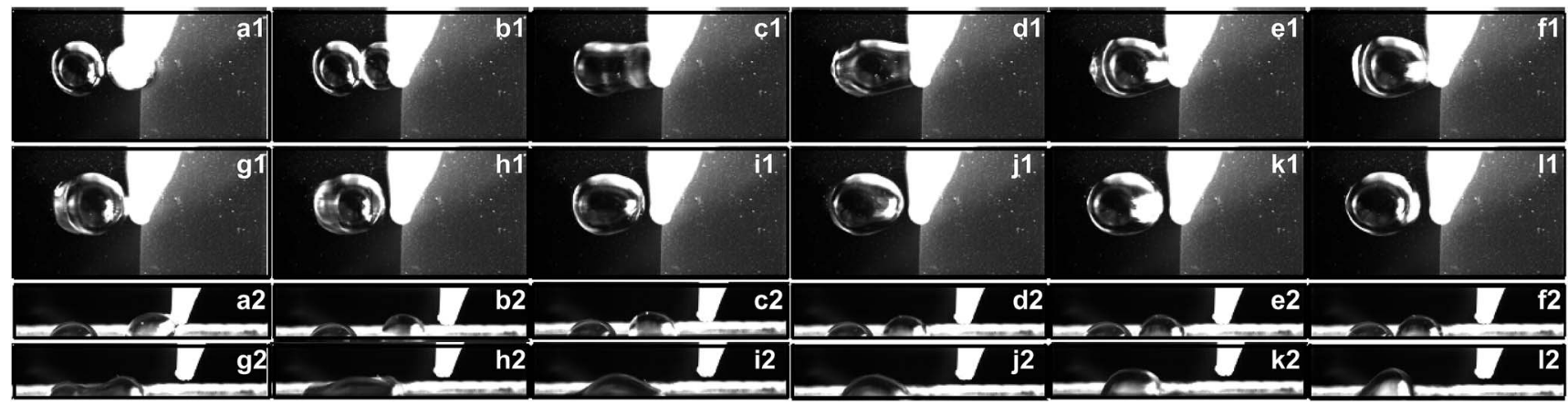

Fig. 4 Coalescence process of one $5 \mu \mathrm{L}$ droplet with another $5 \mu \mathrm{L}$ droplet on specimen I, taken with focus on the top view (a1-l1) and side view (a2-l2): (a1) $0 \mathrm{~s}$; (b1) $0.012 \mathrm{~s}$; (c1) $0.014 \mathrm{~s}$; (d1) $0.016 \mathrm{~s}$; (e1) $0.018 \mathrm{~s}$; (f1) $0.020 \mathrm{~s}$; (g1) $0.022 \mathrm{~s}$; (h1) $0.024 \mathrm{~s}$; (i1) $0.026 \mathrm{~s}$; (j1) $0.028 \mathrm{~s}$; (k1) $0.030 \mathrm{~s}$; (l1) $0.032 \mathrm{~s}$; (a2) $0 \mathrm{~s}$; (b2) $0.014 \mathrm{~s}$; (c2) $0.016 \mathrm{~s}$; (d2) $0.017 \mathrm{~s}$; (e2) $0.020 \mathrm{~s}$; (f2) $0.024 \mathrm{~s}$; (g2) $0.031 \mathrm{~s}$; (h2) $0.032 \mathrm{~s}$; (i2) $0.033 \mathrm{~s}$; (j2) $0.034 \mathrm{~s}$; (k2) $0.035 \mathrm{~s}$; (l2) $0.036 \mathrm{~s}$.

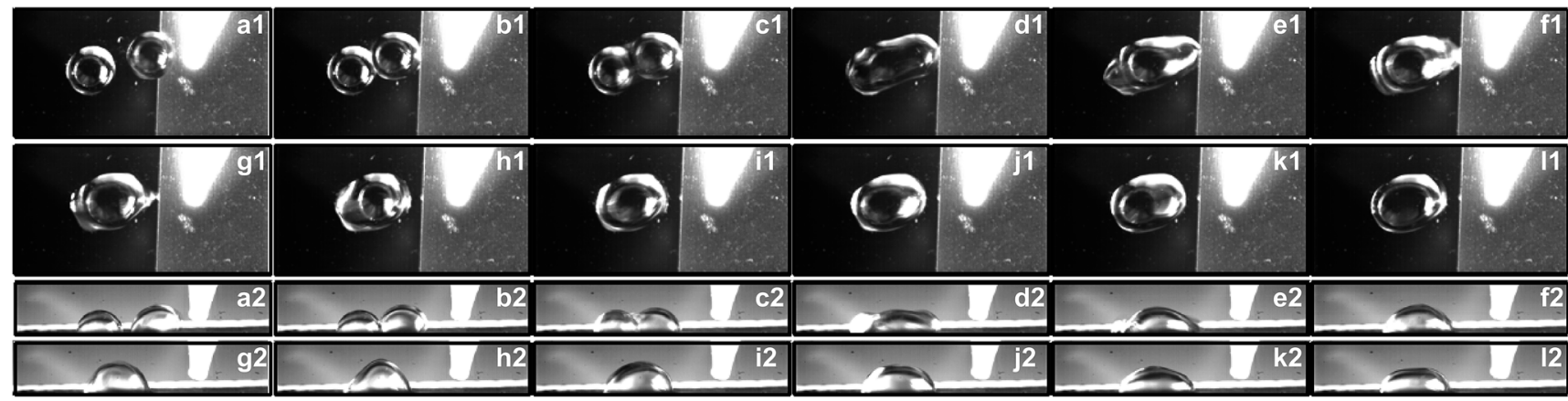

Fig. 5 Coalescence process of one $7 \mu \mathrm{L}$ droplet with a $5 \mu \mathrm{L}$ droplet on specimen II, taken with focus on the top view (a1-l1) and side view (a2-l2): (a1) $0 \mathrm{~s}$; (b1) $0.012 \mathrm{~s}$; (c1) $0.014 \mathrm{~s}$; (d1) $0.016 \mathrm{~s}$; (e1) $0.018 \mathrm{~s}$; (f1) $0.020 \mathrm{~s}$; (g1) $0.022 \mathrm{~s}$; (h1) $0.024 \mathrm{~s}$; (i1) $0.026 \mathrm{~s}$; (j1) $0.028 \mathrm{~s}$; (k1) $0.030 \mathrm{~s}$; (l1) $0.032 \mathrm{~s}$; (a2) $0 \mathrm{~s}$; (b2) $0.010 \mathrm{~s}$; (c2) $0.018 \mathrm{~s}$; (d2) $0.020 \mathrm{~s}$; (e2) $0.022 \mathrm{~s}$; (f2) $0.024 \mathrm{~s}$; (g2) $0.026 \mathrm{~s}$; (h2) $0.028 \mathrm{~s}$; (i2) $0.030 \mathrm{~s}$; (j2) $0.032 \mathrm{~s}$; (k2) $0.034 \mathrm{~s}$; (l2) $0.036 \mathrm{~s}$.

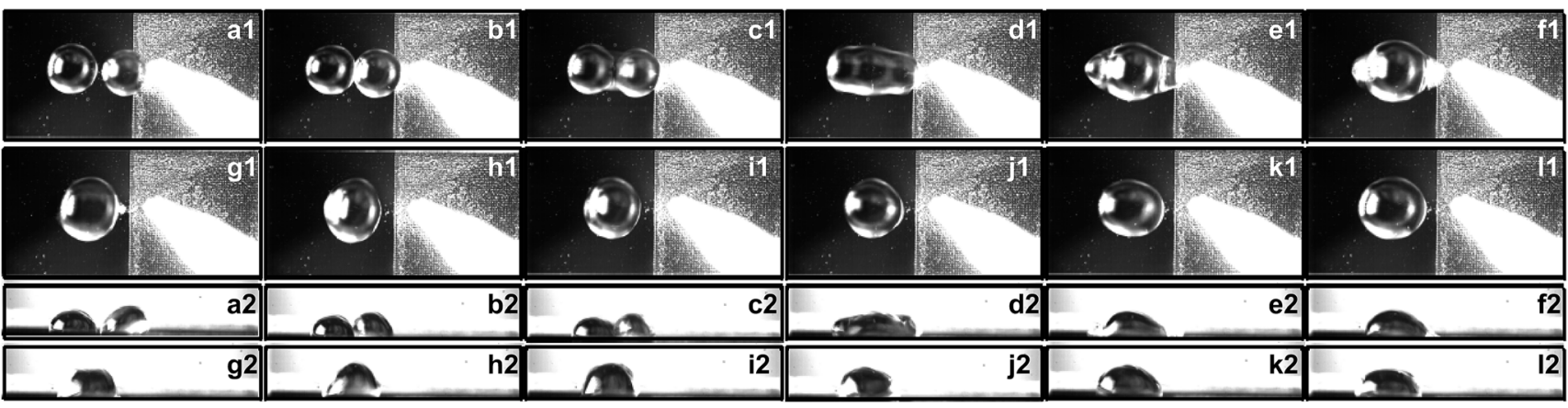

Fig. 6 Coalescence process of one $9 \mu \mathrm{L}$ droplet with a $5 \mu \mathrm{L}$ droplet on specimen III taken with focus on the top view (a1-l1) and side view (a2-l2): (a1) $0 \mathrm{~s}$; (b1) $0.028 \mathrm{~s}$; (c1) $0.029 \mathrm{~s}$; (d1) $0.030 \mathrm{~s}$; (e1) $0.031 \mathrm{~s}$; (f1) $0.032 \mathrm{~s}$; (g1) $0.033 \mathrm{~s}$; (h1) $0.034 \mathrm{~s}$; (i1) $0.035 \mathrm{~s}$; (j1) $0.036 \mathrm{~s}$; (k1) $0.037 \mathrm{~s}$; (l1) $0.038 \mathrm{~s}$; (a2) $0 \mathrm{~s}$; (b2) $0.012 \mathrm{~s}$; (c2) $0.014 \mathrm{~s}$; (d2) $0.016 \mathrm{~s}$; (e2) $0.018 \mathrm{~s}$; (f2) $0.020 \mathrm{~s}$; (g2) $0.022 \mathrm{~s}$; (h2) $0.024 \mathrm{~s}$; (i2) $0.026 \mathrm{~s}$; (j2) $0.028 \mathrm{~s}$; (k2) $0.030 \mathrm{~s}$; (l2) $0.032 \mathrm{~s}$.

When the volume of the approaching droplet increases, the movement of the droplet is faster. However, the final distance before droplet coalescence is larger as the approaching droplet is wider. For the same size of droplet, the spreading of the liquid on the surface is determined by the solid-gas, liquid-gas, and liquid-solid interface tensions. As a result, the base diameter of the droplet remains almost constant for the different cases. The capillary forces vary as the receding contact angles are different.
Based on the study of the contact angle variation for the different specimens, which is presented in Fig. 11 and 12, the increment in pillar intervals enhances the hydrophobicity of the substrate. As a result, the structure of specimen III makes the moving droplet approach the other one faster in order to realize the coalescence process. For a larger droplet, the base diameter increases accordingly, thus the capillary force promotes the motion of the approaching droplet. 


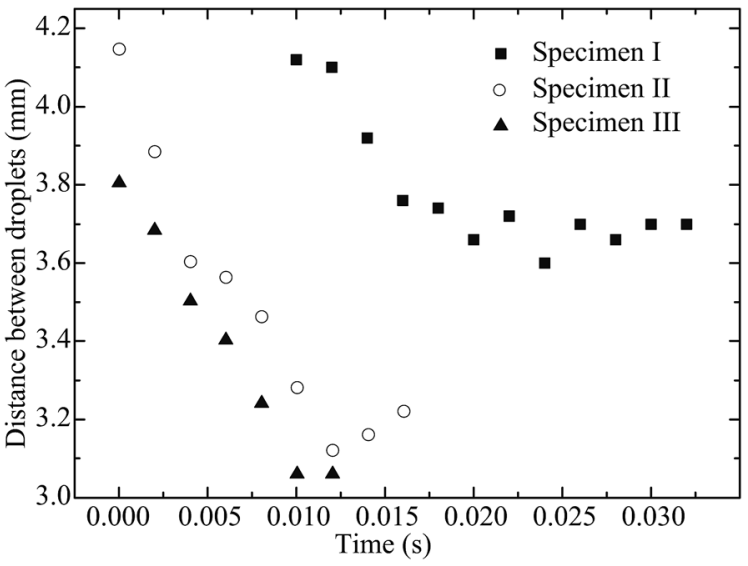

Fig. 7 Distance between two droplets (a $5 \mu \mathrm{L}$ droplet approaching a 5 $\mu \mathrm{L}$ stationary droplet) on solid surfaces with different surface patterns.

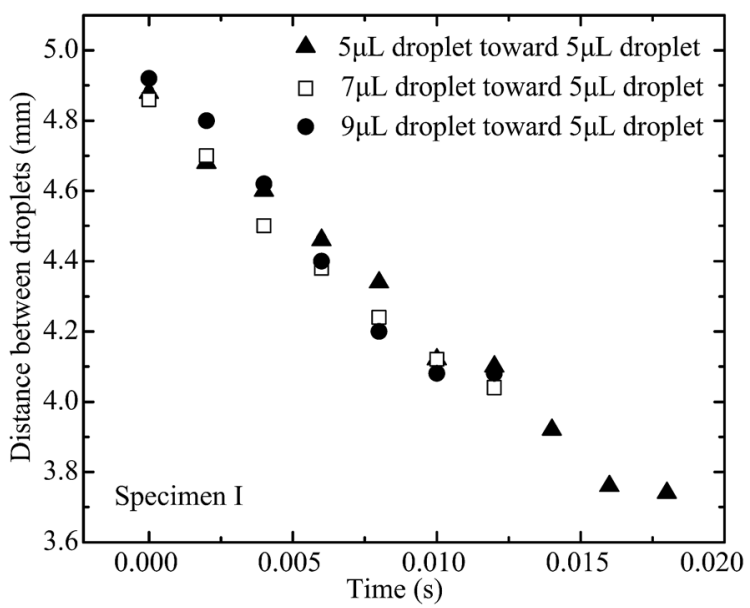

Fig. 8 Distance between two droplets on specimen I with the volume of the moving droplet varying from $5 \mu \mathrm{L}$ to $9 \mu \mathrm{L}$.

\section{Discussion}

In order to obtain a better understanding of the droplet coalescence process, a fundamental investigation is carried out on the wetting properties of deionized water droplets on the patterned substrates. Droplets with different volumes are investigated in the experiments. In a static investigation, the droplet is dispensed on the patterned portion of the surface. The images of the droplets are taken for further analysis. The static contact angle of the water droplet is measured to provide the wetting condition. For comparison, the wetting of the water droplet is first studied on a silicon substrate with homogenous deposition of the photoresist layer. Based on the understanding of the Cassie-Baxter model, the increase of roughness due to the pillars would definitely increase the water contact angle on a patterned surface. A sessile-drop technique is used for the contact angle measurements using a Theta Optical Tensiometer, a versatile system that is able to provide accurate measurements of static and dynamic contact angles, surface

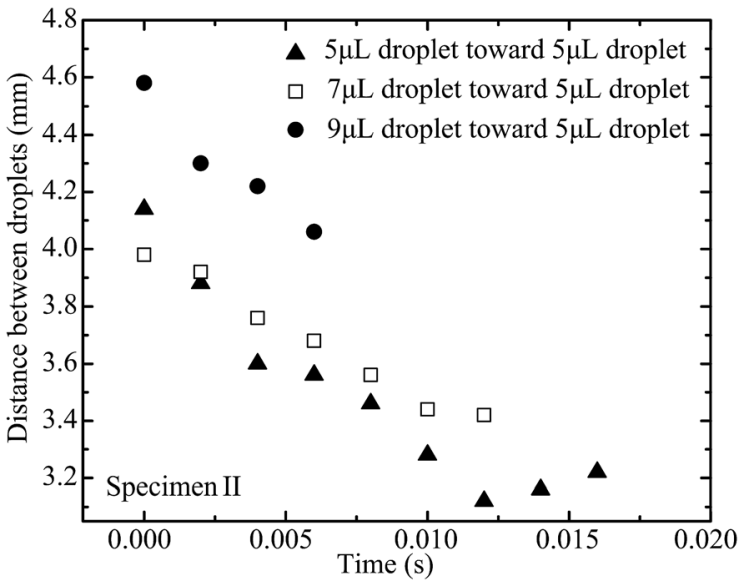

Fig. 9 Distance between two droplets on specimen II with the volume of the moving droplet varying from $5 \mu \mathrm{L}$ to $9 \mu \mathrm{L}$.

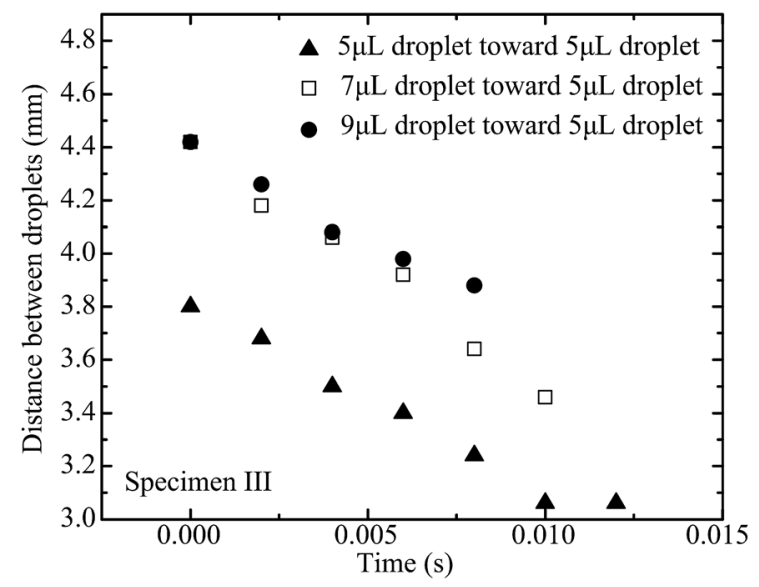

Fig. 10 Distance between two droplets on specimen III with the volume of the moving droplet varying from $5 \mu \mathrm{L}$ to $9 \mu \mathrm{L}$.

free energy, surface and interfacial tension, and interfacial rheology. The liquid dispenser is integrated with a motorized vertical movement, which is fully compatible and controllable with the provided software, and also comes with an automatic single liquid dispenser using disposable pipette tips that can accurately dispense a volume ranging from $0.2 \mu \mathrm{L}$ to $200 \mu \mathrm{L}$.

The wettability of liquids on each specimen has been analyzed according to the contact angles of droplets. The contact angles obtained from all of the specimens are critically assessed for droplet volumes of $5 \mu \mathrm{L}, 7 \mu \mathrm{L}, 9 \mu \mathrm{L}, 12 \mu \mathrm{L}, 15 \mu \mathrm{L}$, and $19 \mu \mathrm{L}$. The data obtained will then be analyzed according to specimens with different pillar intervals. Furthermore, a trend is illustrated to correlate the contact angles with the droplet volumes on the same specimen. For all of the specimens shown in Table 1, the droplet size on the specimens is fixed in order to examine how the pillar intervals affect the contact angles. The experimental results are given for a droplet with a volume at 7 $\mu \mathrm{L}$. Initially, as shown in Fig. 11, the left and right contact angles of the droplet sitting on a flat homogeneous surface coated with AZ-9260 are measured to be $120.78^{\circ}$ and $121.94^{\circ}$, 


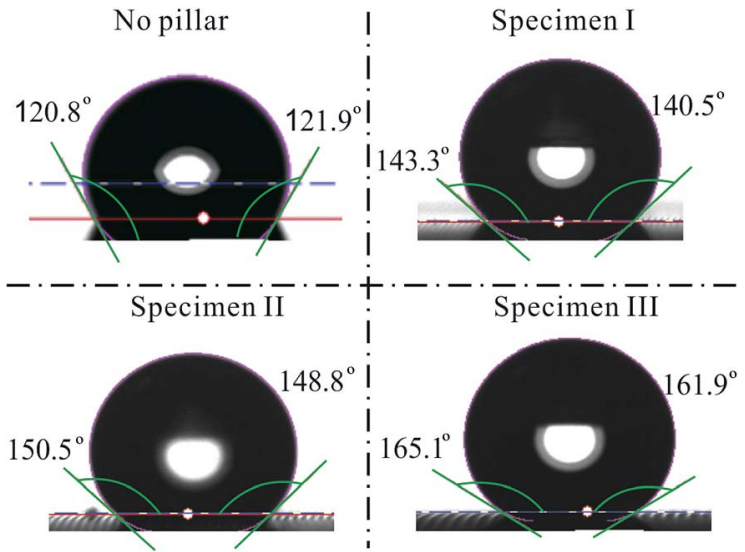

Fig. 11 Contact angle for the $7 \mu \mathrm{L}$ droplet sitting on the flat photoresist layer and specimens I, II, and III.

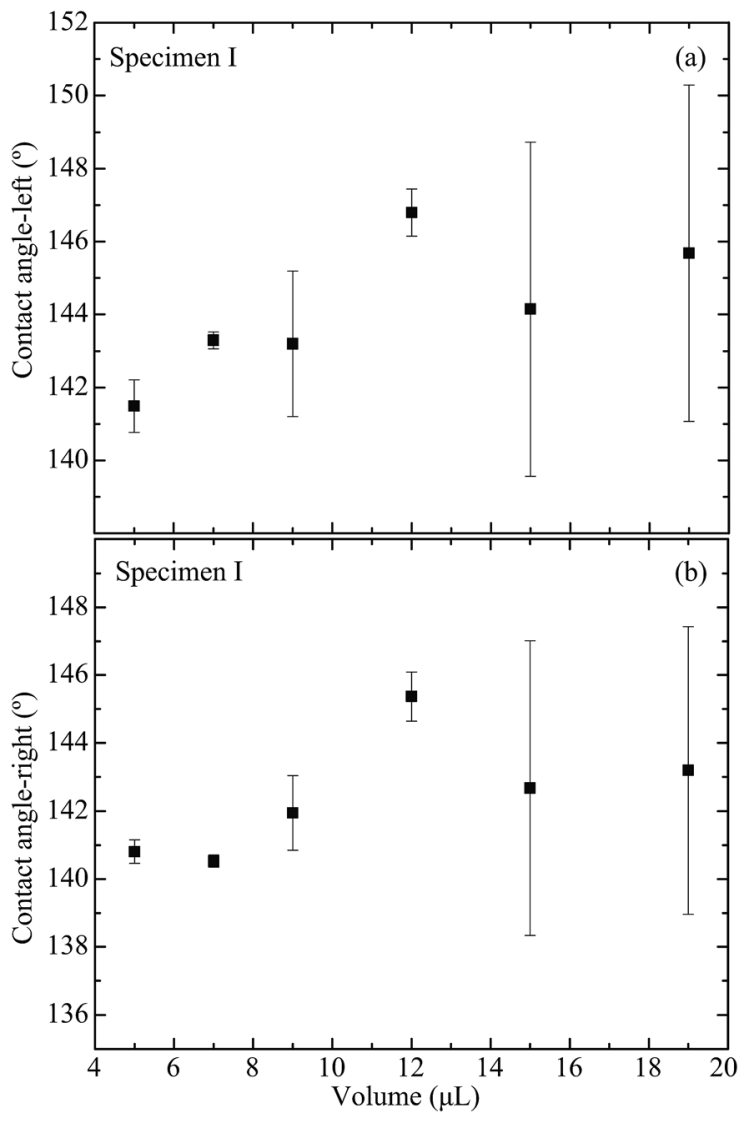

Fig. 12 Left and right contact angles for different volumes of droplets on specimen $\mathrm{I}$.

respectively. The left and right contact angles vary with different pillar intervals. As shown in Fig. 11, the left contact angles of specimens I, II, and III are $143.29 \pm 0.23^{\circ}, 150.53 \pm 1.48^{\circ}$, and $165.11 \pm 0.43^{\circ}$, respectively. The right contact angles of specimens I, II, and III are $140.53 \pm 0.18^{\circ}, 148.83 \pm 0.81^{\circ}$, and 161.87 $\pm 0.61^{\circ}$, respectively. As a result, the pillar structure makes the surface have a higher hydrophobicity. A larger pillar interval increases the contact angle of a water droplet sitting on the surface. The change in the contact angle can be explained based on the Cassie-Baxter wetting model, following the equation

$$
\cos \theta^{*}=r_{\mathrm{f}} f \cos \theta_{\mathrm{y}}+f-1,
$$

where $r_{\mathrm{f}}$ is the roughness ratio of the wetted surface area, $f$ is the fraction of solid surface area wetted by the liquid, $\theta^{*}$ is the apparent contact angle which is the subject of interest, and $\theta_{\mathrm{y}}$ is the original contact angle of a real fraction of the composite material. The roughness ratio of the specimens' surfaces can be measured using a Talyscan 150 by Taylor Hobson with an inductive gauge. The value of the initial contact angle $\theta_{\mathrm{y}}$ is obtained by measuring the contact angle with the Theta Optical Tensiometer on the silicon wafer that is modified only with a layer of the chemical AZ-9260 without the additional modification of pillars. The Cassie-Baxter model states that liquid would only come into contact with the heterogeneous top layer and leave air below the uneven surface. In the Cassie-Baxter equation, the fraction of the solid surface area wetted by the liquid, $f$, is one of the important factors that can affect the contact angle. With an increase of the pillar interval, the fraction of the solid surface area wetted by the liquid would decrease accordingly. The combination of the changes in $r_{\mathrm{f}}$ and $f$ induces a surface wettability shift.

In addition, the effect of the liquid volume on the water droplet's wetting behavior is studied. For specimens I, II, and III, all of the different volumes demonstrate the rather consistent result of having an increase of the contact angle with the increase of the pillar interval. To investigate the effect of different droplet volumes on the same specimen, the experimental data are analyzed; the contact angles vary with the droplet volume. Fig. 12(a) and (b) present the effect of droplet volume on the specimen I wetting properties indicated by the left and right contact angles, respectively. This indicates a low influence on the correlation between the droplet volume and the contact angle in the range of micro-liter droplets.

\section{Conclusions}

The contact angles of droplets on specimens I, II, and III were obtained experimentally by varying the droplet volume on all the specimens. The collected contact angles were summarized in order to analyze the factors affecting the contact angle. Specimens I, II, and III had an increasing pillar interval and displayed a corresponding trend of increasing contact angle. The effect of the droplet volume on the different specimens was also investigated. The contact angles on all the specimen surfaces were observed to be independent of the variation of the droplet volumes. Droplet width was investigated for the dependence on the droplet volume. The capillary force generated by a droplet moving over a transition line of wettability was used as an actuation mechanism to induce movement, which eventually led to the coalescence of two droplets. The effects of the different pillar intervals, contact angles, and droplet volumes on the capillary force were discussed. The results showed that specimens with a higher contact angle (more hydrophobic) and a larger droplet volume produced higher 
capillary forces. Similarly, an increase in the droplet volume had a direct influence on the length of the droplet base diameter, hence increasing the capillary force.

\section{Acknowledgements}

This work was partially supported by the Fundamental Research Funds for the Central Universities, No. NS2016086.

\section{References}

1 V. S. Nikolayev, D. Beysens and P. Guenoun, Phys. Rev. Lett., 1996, 76, 3144-3147.

2 L. Duchemin, J. Eggers and C. Josserand, J. Fluid Mech., 2003, 487, 167-178.

3 S. K. Cho, H. Moon and C.-J. Kim, J. Microelectromech. Syst., 2003, 12, 70-80.

4 I. A. Aksay, C. E. Hoge and J. A. Pask, J. Phys. Chem., 1974, 78, 1178-1183.

5 S. David, K. Sefiane and L. Tadrist, Colloids Surf., A, 2007, 298, 108-114.

6 T. H. Ting, Y. F. Yap, N. T. Nguyen, T. N. Wong, J. C. K. Chai and L. Yobas, Appl. Phys. Lett., 2006, 89, 234101.

7 M. Chakraborty, U. U. Ghosh, S. Chakraborty and S. DasGupta, RSC Adv., 2015, 5, 45266.

8 J. M. Galvin and G. B. Schuster, Supramol. Sci., 1998, 5, 89100.

9 R. Rosario, D. Gust, M. Hayes, F. Jahnke, J. Springer and A. A. Garcia, Langmuir, 2002, 18, 8062-8069.

10 R. A. Hayes and B. Feenstra, Nature, 2003, 425, 383-385.

11 U. C. Yi and C. J. Kim, J. Micromech. Microeng., 2006, 16, 2053-2059.

12 N. T. Nguyen, G. Zhu, Y. C. Chua, V. N. Phan and S. H. Tan, Langmuir, 2010, 26, 12553-12559.

13 R. E. Rosensweig, Ferrohydrodynamics, Cambridge University Press, New York, 1985.

14 J. J. Bikerman, J. Colloid Sci., 1950, 5, 349-359.

15 S. Moghtadernejad, M. Tembely, M. Jadidi, N. Esmail and

A. Dolatabadi, Phys. Fluids, 2015, 27, 032106.

16 C. Furmidge, J. Colloid Sci., 1962, 17, 309-324.

17 K. Kawasaki, J. Colloid Sci., 1960, 15, 402-407.

18 B. J. Briscoe and K. P. Galvin, Colloids Surf., 1991, 52, 219229.

19 D. Quere, M.-J. Azzopardi and L. Delattre, Langmuir, 1998, 14, 2213-2216.

20 M. Ahmadlouydarab and J. J. Feng, J. Fluid Mech., 2014, 746, 214-235.
21 J. Hyvaluoma, A. Koponen, P. Raiskinmaki and J. Timonen, Eur. Phys. J. E, 2007, 23, 289-293.

22 J. Lv, Y. Song, L. Jiang and J. Wang, ACS Nano, 2014, 8, 31523169.

23 K. Li, S. Xu, W. Shi, M. He, H. Li, S. Li, X. Zhou, J. Wang and Y. Song, Langmuir, 2012, 28, 10749.

24 Q. Zhang, M. He, X. Zeng, K. Li, D. Cui, J. Chen, J. Wang, Y. Song and L. Jiang, Soft Matter, 2012, 8, 8285-8288.

25 Q. Zhang, M. He, J. Chen, J. Wang, Y. Song and L. Jiang, Chem. Commun., 2013, 49, 4516-4518.

26 R. Dou, J. Chen, Y. Zhang, X. Wang, D. Cui, Y. Song, L. Jiang and J. Wang, ACS Appl. Mater. Interfaces, 2014, 6, 6998.

27 J. Chen, R. Dou, D. Cui, Q. Zhang, Y. Zhang, F. Xu, X. Zhou, J. Wang, Y. Song and L. Jiang, ACS Appl. Mater. Interfaces, 2013, 5, 4026-4030.

28 J. Chen, J. Liu, M. He, K. Li, D. Cui, Q. Zhang, X. Zeng, Y. Zhang, J. Wang and Y. Song, Appl. Phys. Lett., 2012, 101, 111603.

29 G. Faeth, Prog. Energy Combust. Sci., 1977, 3, 191-224.

30 J. Eggers, J. R. Lister and H. A. Stone, J. Fluid Mech., 1999, 401, 293-310.

31 D. G. A. L. Aarts, H. N. W. Lekkerkerker, H. Guo, G. H. Wegdam and D. Bonn, Phys. Rev. Lett., 2005, 95, 164503.

32 J. Qian and C. Law, J. Fluid Mech., 1997, 331, 59-80.

33 K. Hosokawa, T. Fujii, and I. Endo, Proceedings of the IEEE Micro Electro Mechanical Systems (MEMS), IEEE, Piscataway, NJ, United States, 1999, pp. 388-393.

34 K. Hosokawa, T. Fujii and I. Endo, Anal. Chem., 1999, 71, 4781-4785.

35 S. K. Cho, H. Moon and C. J. Kim, J. Microelectromech. Syst., 2003, 12, 70-80.

36 W. D. Ristenpart, P. M. McCalla, R. V. Roy and H. A. Stone, Phys. Rev. Lett., 2006, 97, 064501.

37 J. A. Diez and L. Kondic, J. Comput. Phys., 2002, 183, 274-306.

38 H. Wang, X. Zhu, Q. Liao and P. Sui, J. Supercond. Novel Magn., 2010, 23, 1137-1140.

39 C. Andrieu, D. Beysens, V. Nikolayev and Y. Pomeau, J. Fluid Mech., 2002, 453, 427-438.

40 R. Narhe, D. Beysens and V. S. Nikolayev, Langmuir, 2004, 20, 1213-1221.

41 N. Kapur and P. H. Gaskell, Phys. Rev. E: Stat., Nonlinear, Soft Matter Phys., 2007, 75, 056315.

42 R. Narhe, D. Beysens and V. S. Nikolayev, Int. J. Thermophys., 2005, 26, 1743-1757. 